\title{
Diffusion Tensor Imaging Properties and Neurobehavioral Outcomes in Children with Hydrocephalus
}

\author{
W. Yuan, R.C. McKinstry, J.S. Shimony, M. Altaye, S.K. Powell, J.M. Phillips, D.D. Limbrick, Jr,
} S.K. Holland, B.V. Jones, A. Rajagopal, S. Simpson, D. Mercer, and F.T. Mangano

\begin{abstract}
BACKGROUND AND PURPOSE: White matter structural alterations and the correlation with neuropsychological deficits in children with hydrocephalus have not been well investigated. In this prospective study, the objectives were the following: 1) to apply DTI to detect in vivo white matter alterations based on diffusion properties in children with acute hydrocephalus, 2) to quantify early neuropsychological deficits, and 3) to explore the correlation between potential neuropsychological deficits and abnormalities in functionally related white matter.
\end{abstract}

MATERIALS AND METHODS: A total of 44 children, 24 with hydrocephalus and 20 controls, were enrolled in the study. DTI indices, FA, $M D, A D$, and RD, were evaluated in the gCC, SCC, PLIC, and ALIC. The ABAS-II was used as a broad screener of development, including conceptual, social, practical, and motor skills. The correlation between the Motor Scale and DTI indices in the PLIC was analyzed.

RESULTS: DTI analyses showed that the gCC and SCC in children with hydrocephalus had lower FA and higher MD, driven by the increased RD with statistical significance $(P<.05)$ or trend-level significance $(P=.06)$. The PLIC and ALIC had significantly higher AD in children with hydrocephalus $(P<.05)$. On the ABAS-II, parent ratings of general adaptive skills, conceptual skills, and motor skills were significantly lower in children with hydrocephalus (all at $P<.05$ ). The MD and RD values in the PLIC were found to have trend-level or significant correlation with the Motor Scale $(P=.057, .041$, respectively).

CONCLUSIONS: DTI reveals alterations in the white matter structure in children with hydrocephalus with preliminary findings suggesting correlation with clinical motor deficits.

ABBREVIATIONS: ABAS-II = Adaptive Behavior Assessment System, Second Edition; AD = axial diffusivity; ALIC = anterior limb of internal capsule; $F A=$ fractional anisotropy; $\mathrm{gCC}=$ genu of the corpus callosum; IVH = intraventricular hemorrhage; $\mathrm{MD}=$ mean diffusivity; $\mathrm{PLIC}=$ posterior limb of the internal capsule; $\mathrm{RD}=$ radial diffusivity; sCC = splenium of the corpus callosum

$\mathrm{H}$ ydrocephalus is a pathologic imbalance in the production and absorption of CSF. The resulting increased intracranial pressure can cause extensive structural displacement, mechanical distortion, and eventually injury throughout the brain. ${ }^{1}$ The resulting damage to the corpus callosum, periventricular white matter, fornix, internal capsule, and other white matter structures has been consistently reported to be a major factor associated with

Received March 22, 2012; accepted after revision April 9.

From the Department of Radiology (W.Y., S.K.H., B.V.J., A.R., S.S.), Division of Biostatistics and Epidemiology (M.A.), Division of Developmental and Behavioral Pediatrics-Psychology (I.M.P.), and Division of Pediatric Neurosurgery (F.T.M.), Cincinnati Children's Hospital Medical Center, Cincinnati, Ohio; University of Cincinnati College of Medicine (W.Y., M.A., J.M.P., S.K.H., B.V.J., F.T.M.), Cincinnati, Ohio; Mallinckrodt Institute of Radiology (R.C.M., J.S.S.), Department of Neurology (S.K.P.), Department of Neurological Surgery (D.D.L., D.M.), and Washington University School of Medicine (R.C.M., J.S.S., S.K.P., D.D.L., D.M.), Washington University, St. Louis, Missouri; and Department of Psychology (S.K.P.), St. Louis Children's Hospital, St. Louis, Missouri.

This work was supported by grant National Institutes of Health/National Institute of Neurological Disorders and Stroke 1R01 NS066932. poor neurologic outcomes. ${ }^{1-6}$ However, despite increasing recognition of the role of white matter injury in hydrocephalus-related neurologic challenges, the pathophysiology remains poorly understood. Conventional MR imaging techniques, such as T1WI or T2WI, offer the capability to detect major structural abnormalities but lack the sensitivity and/or specificity to quantify white matter abnormalities in patients with hydrocephalus. ${ }^{7,8}$

DTI is an advanced MR imaging technique that quantifies the microscopic diffusion properties of water molecules in biologic tissues. These diffusion parameters can be used to characterize the degree of organization of the neural tissue in the brain. In patho-

The data reported in this manuscript have not been presented or published elsewhere.

Please address correspondence to Francesco T. Mangano, DO, Division of Pediatric Neurosurgery, Cincinnati Children's Hospital, MLC 2016, 3333 Burnet Ave, Cincinnati, $\mathrm{OH}$ 45229; e-mail: francesco.mangano@cchmc.org

- Indicates open access to non-subscribers at www.ajnr.org

三 Indicates article with supplemental on-line tables.

http://dx.doi.org/10.3174/ajnr.A3218 
logic conditions causing structural abnormalities or tissue degeneration, DTI measurements can quantify these changes and can be used as a noninvasive imaging biomarker to monitor disease progression or therapeutic efficacy; furthermore, DTI may have a unique role in determining neurologic prognosis. In recent studies of hydrocephalus, DTI has shown white matter anisotropic changes in hydrocephalus as well as postoperative normalization in successfully treated children. ${ }^{9-13}$ However, these studies were often small in size and retrospective in nature or included a heterogeneous population of children with hydrocephalus.

The purpose of this study was to prospectively quantify the diffusion properties in white matter structures of a group of children presenting with the age range and clinical profiles typical of pediatric hydrocephalus. We hypothesized that children with hydrocephalus would demonstrate abnormal diffusion properties that may be characteristically different in the corpus callosum and internal capsule and that neuropsychological outcome measures would be abnormal and correlated with the DTI measurements.

\section{MATERIALS AND METHODS}

All participants were selected from an ongoing multicenter, prospective, longitudinal imaging study of pediatric hydrocephalus. The present study reports initial results derived from imaging and neurobehavioral tests at baseline before surgical management.

Participants. A total of 44 participants, 24 children with hydrocephalus (age range, 0-51 months) and 20 healthy control children (age range, 3-68 months) were recruited. Among the 24 patients with hydrocephalus, 3 had communicating hydrocephalus, 1 had unknown etiology, and the remaining 20 had obstructive hydrocephalus including different etiologies: aqueduct stenosis $(n=7)$ IVH $(n=4)$, posterior fossa or arachnoid cyst $(n=4)$, and other etiologies $(n=5)$. DTI and ABAS-II ${ }^{14}$ data were acquired from 15 and 17 patients, respectively. Two of the 15 patients with hydrocephalus on DTI had severe hydrocephalus and ventriculomegaly, which did not allow reliable delineation of any region of interest. Among the 20 healthy participants, 2 children were found to have standard scores below 80 across all 4 ABAS-II domains, as well as the motor subdomain, and thus were disqualified and excluded from further analyses. DTI data were acquired from 17 of the remaining 18 healthy participants; ABAS-II data were acquired from 16 . The final region of interest-based group comparisons of DTI indices involved 13 patients with hydrocephalus (mean age, 10 months; range, $0-39$ months) and 17 controls (mean age, 34 months; range, 3-68 months). The group comparisons of ABAS-II scores involved 17 patients with hydrocephalus (mean age, 17 months; range, 0-51 months) and 16 healthy controls (mean age, 35 months; range, 3-68 months). The correlation analysis included 8 children with hydrocephalus (5 of the 13 patients with hydrocephalus described above did not have an ABAS-II test). Detailed demographic information for the control and patient groups are listed in On-line Tables 1 and 2, respectively.

Two hospitals, Cincinnati Children's Hospital Medical Center and St. Louis Children's Hospital, were the sites of recruitment. Parents gave consent when participants were enrolled into the study according to institutional review board guidelines at both institutions. Inclusion criteria for the hydrocephalus group were the following: 1) age, $0-15$ years, 2) referral to radiology for brain MR imaging for evaluation of hydrocephalus and later referral for CSF diversion surgery, 3) hydrocephalus with ventriculomegaly, and 4) no evidence of other medical diagnoses that would predispose the child to adverse neurologic outcomes (eg, preterm birth with severe intraventricular hemorrhage, Dandy-Walker syndrome, meningitis, stroke, or spina bifida). Inclusion criteria for the control group were the following: 1) age, 0-16 years, 2) referral to radiology for nonspecific symptoms not clearly related to a neurologic disorder (eg, headaches), 3) diagnosis of normal MR imaging findings, 4) no clinical or radiographic history of neurologic or psychological disorders before the scan, and 5) no evidence of any neurologic disorders (eg, epilepsy, stroke) or suggestion of white matter-related brain pathology within 3 months after the initial MR imaging per medical records.

MR Imaging/DTI Data Acquisition and Processing. DTI data were acquired as part of a clinical scan on $1.5 \mathrm{~T}$ scanners at either Cincinnati Children's Hospital Medical Center (Signa; GE Healthcare, Milwaukee, Wisconsin) or St. Louis Children's Hospital (Avanto; Siemens, Erlangen, Germany). A diffusion-weighted spin-echo sequence with single-shot EPI was used with the following specifications: $\mathrm{FOV}=240 \times 240 \mathrm{~mm}$, matrix $=96 \times 96$, in-plane resolution $=2.5 \times 2.5 \mathrm{~mm}$, section thickness $=2.5 \mathrm{~mm}$, number of sections $=76, \mathrm{TR} / \mathrm{TE}=9400 / 93.2 \mathrm{~ms}$, array spatial sensitivity encoding technique or integrated parallel acquisition technique factor $=2$. Diffusion weighting was applied with $b=1000 \mathrm{~s} / \mathrm{mm}^{2}$ along 15 independent noncollinear orientations. One additional image with no diffusion weighting $(b=0)$ was also acquired. Two averages were used to increase the SNR. Highresolution sagittal T1-weighted images were also acquired $(1 \times$ $1 \times 1 \mathrm{~mm}^{3}$ ) for assessing ventricle size on the basis of the fronto-occipital horn ratio.

Image processing and analysis were performed by using the DTIStudio 3.02 software (Johns Hopkins University, Baltimore, Maryland), ${ }^{15}$ in which the automated image-registration method was used to correct for head motion and eddy current artifacts. ${ }^{16}$ An automatic bad-sector detection method and additional visual inspection were used to identify signal drop-out on DWI. DTI metrics, including $\mathrm{FA}, \mathrm{MD}, \mathrm{AD}$, and $\mathrm{RD}$, were calculated by using the standard technique. ${ }^{17}$ The ROIs included the gCC, sCC, ALIC, and PLIC. Delineation of ROIs was based on the approach of Hermoye et al, ${ }^{18}$ which was also used in our previous work. ${ }^{12,13}$ DTI measurements for ALIC and PLIC were initially calculated bilaterally and were averaged, because no significant difference was found between the 2 hemispheres.

Quality Assurance in Multicenter and Multiscanner Neuroimaging Research. To establish the MR imaging compatibility across the 2 sites (two 1.5T Signa scanners from Cincinnati Children's Hospital Medical Center and one 1.5T Avanto from St. Louis Children's Hospital), we tested the complete MR imaging protocol, including DTI, T1WI, and T2WI sequences, at both sites by using the same MR imaging phantom (Functional Bioinformatics Research Network, http://www.na-mic.org/Wiki/index.php/ FBIRN:Main_Page) and the same human subject before the start of the study; this process has been repeated annually since. The protocol also requires both sites to collect quality assurance data 

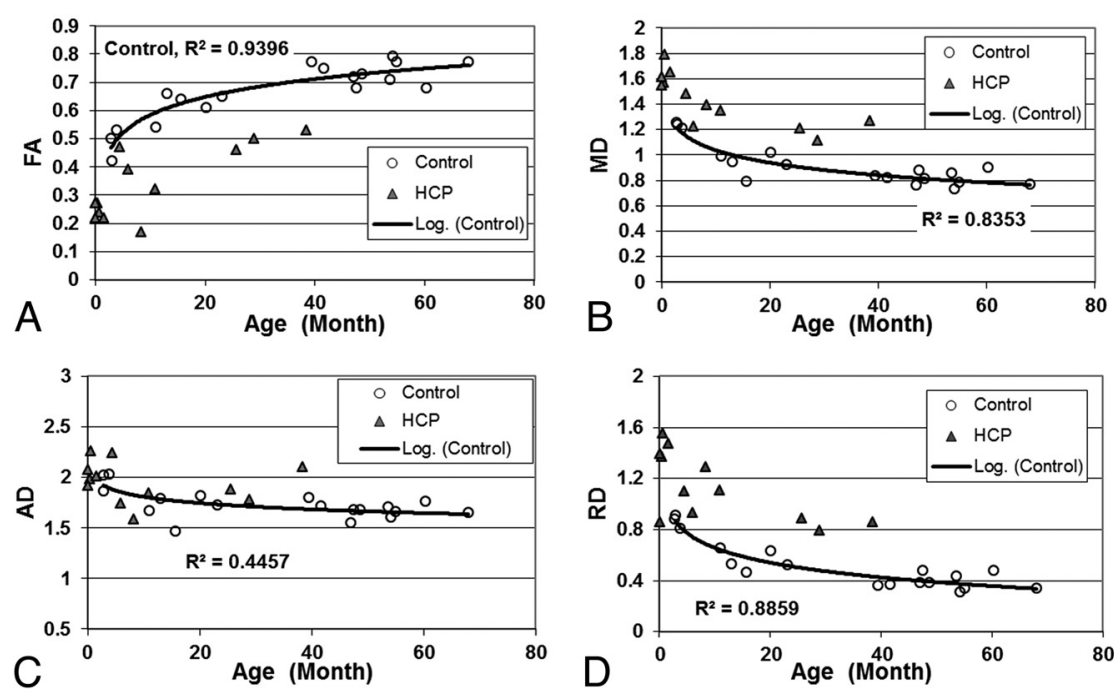

FIG 1. Scatterplot showing the group difference of $(A) F A ;(B) M D ;(C) A D ;$ and $(D)$ RD in the gCC.
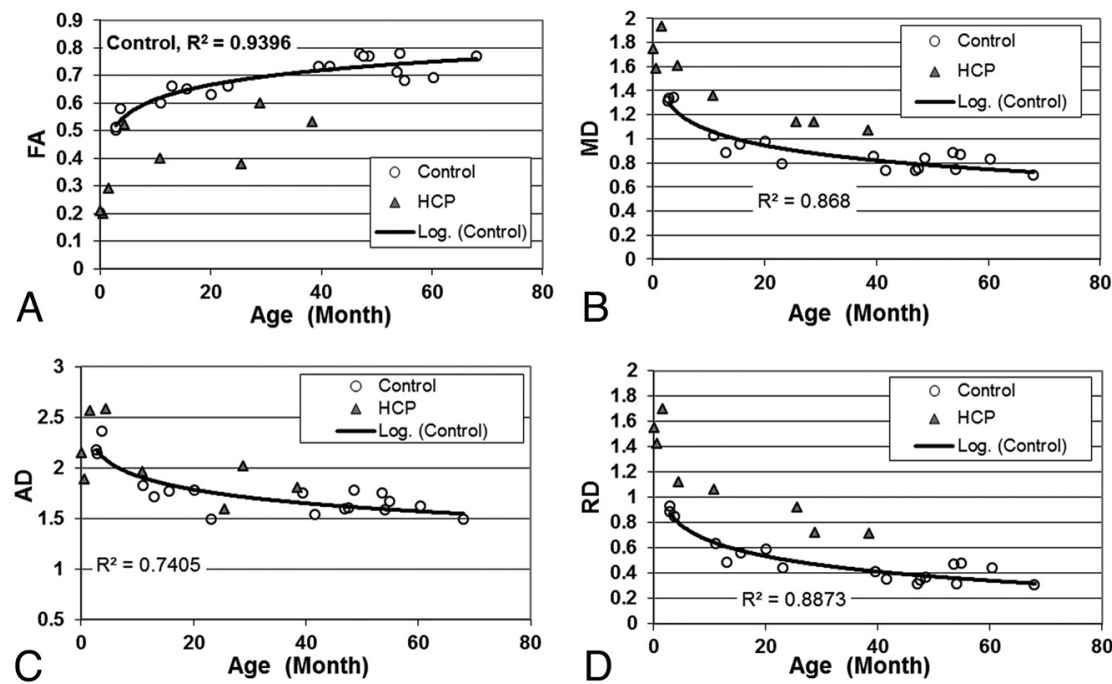

FIG 2. Scatterplot showing the group difference of (A)FA; (B) MD; (C) AD; and (D) RD in the SCC.

by using American College of Radiology phantoms within a week of each subject's scan to assess the stability of the scanner performance and to detect system-performance change relative to the established baseline. As reported elsewhere, ${ }^{19}$ the scanners from the 2 sites showed high compatibility during the initial setup; the routine quality assurance scans also showed that scanner performance remained highly stable throughout the period in geometric measurement, SNR, and DTI measurement. It has been shown in several multicenter imaging studies that it is possible to obtain consistent and compatible image quality across sites and scanners if one appropriately attends to details of scanner performance and protocols. ${ }^{20,21}$ In the present study, all the quality assurance procedures described above were followed rigorously and results were up-to-date at the time of data analysis for the study. In addition, the different scanners are also controlled as a source of variability in the statistical analysis model we used. ${ }^{22}$

Neuropsychological Evaluation. A battery of neuropsychological tests was included in the ongoing parent imaging study. The pres- ent report highlights composite scores from the ABAS- $\mathrm{II}^{14}$ and their correlation with imaging results. The ABAS-II is a comprehensive age-normed caregiver-report measure of a child's independence skills, covering specific functional domains, including communication, daily living, and social and motor skills. The results comprise an overall score (General Adaptive Composite) and 3 functional domain scores (Conceptual, Social, and Practical). In addition, there is a Motor Scale independent of the functional domain scales. Parents were asked to complete the ABAS-II shortly after initial diagnosis but before surgical intervention.

Statistical Analysis. Statistical analyses were performed by using SAS 9.2 (SAS Institute, Cary, North Carolina). We initially examined the distribution of data for normality and understanding of the relationship of age with DTI outcomes. Because age and DTI outcomes exhibit an exponential relationship, we transformed the age variable to a log scale to make the relationship linear. Subsequently, we fitted a general linear model to compare the difference between control and hydrocephalus groups after controlling for logtransformed age and potential scanner effect. We also looked at the correlation between DTI and behavioral outcomes by using Spearman correlation coefficients. Before running the correlation analysis, we removed the inherent age effect by fitting a regression model relating DTI outcome with log-transformed age. Examining the distribution of neuropsychological measures plotted against age, a linear relationship was observed for some of the measures. Hence, we removed the age effect from the neuropsychological data by fitting a linear regression model relating ABAS-II scores to age. We then extracted the residuals (difference between fitted and original DTI values) that were free of age effect and correlated these with the residual behavioral outcome by using the Spearman correlation for each group separately.

\section{RESULTS}

The values of the 4 DTI indices of individual participants are displayed in the scatterplots in Figs 1-4. As expected, there was a general trend of increase in $\mathrm{FA}$ with age and in $\mathrm{MD}, \mathrm{AD}$, and $\mathrm{RD}$ with age in the control group. A similar trend was also found in patients with hydrocephalus. The scanner was not found to be a significant factor in any of the comparisons. The significance of age and scanner factors and the group DTI differences with adjustment for age and scanner are listed in Table 1 and described in detail below. Ventricle size (fronto-occipital horn ratio) was 

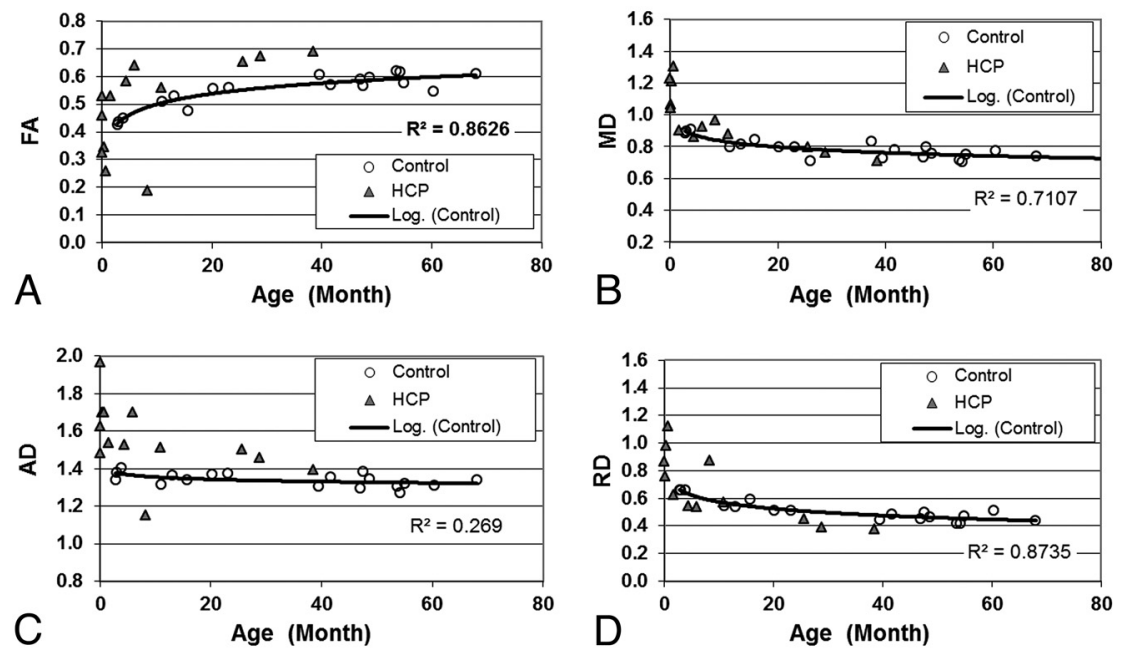

FIG 3. Scatterplot showing the group difference of $(A)$ FA; $(B)$ MD; (C) AD; and (D) RD in the PLIC.
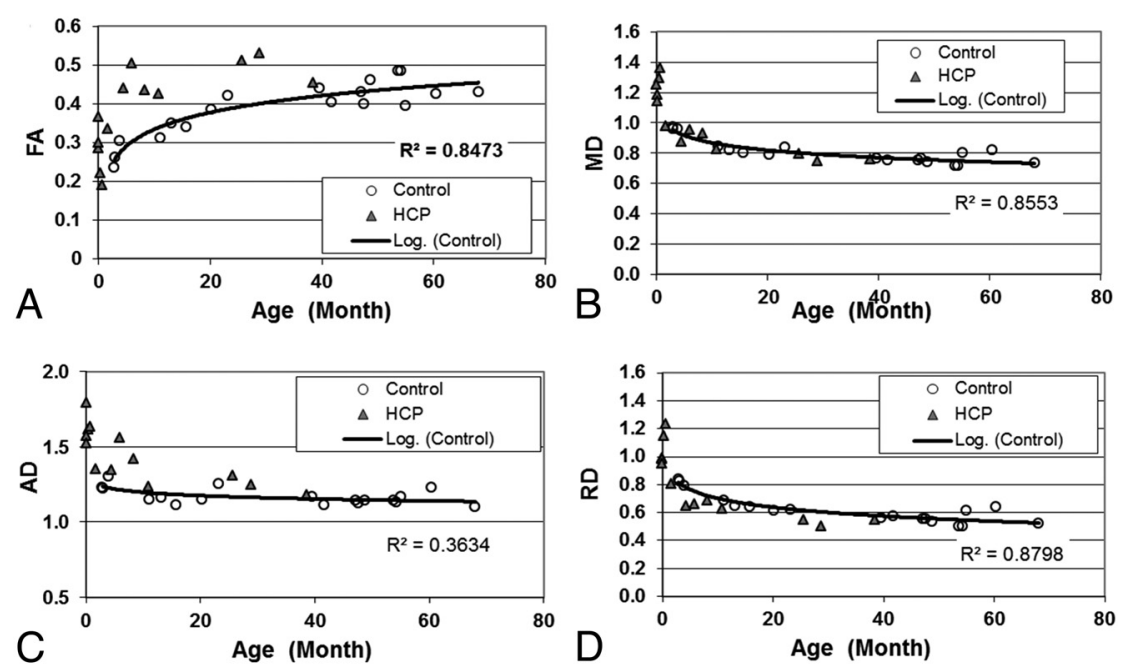

FIG 4. Scatterplot showing the group difference of (A) FA; (B) MD; (C) AD; and (D) RD in the ALIC.

found to be significantly different between the control and patient groups (mean, $0.31 \pm 0.03$ versus $0.59 \pm 0.14 ; P<.0001$ ). No significant correlation was found between fronto-occipital horn ratio and any of the DTI indices.

\section{Abnormal DTI Indices in the Corpus Callosum}

Age was found to influence DTI values with statistical significance in both the gCC and sCC (Table 1). In the gCC (Fig 1), FA values increased with age $(P<.0001$, corrected $)$, while MD and RD values decreased with age $(P<.0001, P<.005$, respectively; both corrected). After adjustment for age and scanner, we found the children with hydrocephalus to have significantly lower FA $(P<.005$, corrected $)$, higher MD $(P<.005$, corrected $)$, and higher $\mathrm{RD}(P<.005$, corrected $)$ in the gCC compared with controls.

In the sCC, a similar influence of age, as well as age- and scanner-adjusted group differences, was found in DTI indices (Fig 2 and Table 1).
Abnormal DTI Indices in the Internal Capsule

Similar to the corpus callosum, age was found to be a significant factor affecting DTI indices for both the ALIC and PLIC (all $P<.005$, corrected; Table 1). Compared with controls, the patients with hydrocephalus had significantly higher AD values in the PLIC $(P<.01$, corrected; Table 1 and Fig 3 ). They also had higher $\mathrm{AD}$ with statistical significance $(P<.005$, corrected $)$ and higher FA with marginal significance $(P=.058$, corrected) in the ALIC (Table 1 and Fig 4).

\section{Abnormal Neuropsychological Outcomes}

As shown in Table 2 and Fig 5, parent response on the ABAS-II yielded significantly lower General Adaptive Composite scores in the hydrocephalus group compared with controls, with significant deficits noted in all the subcategories of the results, which included Conceptual, Practical, Social, and Motor and Motor Scales (all $P<.05$ ).

In the hydrocephalus group, the ABAS-II Motor score was found to have a negative correlation with both $\mathrm{MD}$ and RD residuals in the PLIC after adjusting for age at trend-level significance or with statistical significance (Spearman correlation coefficient $=-0.71$, -0.74 , respectively; $P=.057, .046$, respectively; Fig 6). No significant or trend-level correlations were found between the Motor score and other DTI indices in patients with hydrocephalus or between the Motor score and any DTI indices in the control group.

\section{DISCUSSION}

Long-term, prospective, longitudinal DTI studies of hydrocephalus in children are lacking. The ongoing parent project of this study is the first of its kind, to our knowledge. Its overall goal is to use DTI to evaluate white matter integrity as a noninvasive biomarker to quantify in vivo injury and posttreatment recovery in children with hydrocephalus. The data presented in the current report are the initial results derived from baseline data collected before CSF diversion surgery. Our main findings in this initial study include the following: First, the diffusion properties in children with hydrocephalus are significantly altered in several important white matter regions in the corpus callosum and internal capsule. Second, the patterns of abnormality in DTI measurements vary in different white matter regions. Patients with hydrocephalus were found to have lower FA and higher MD in the gCC 
Table 1: Group difference in the DTI measurement between the patients with HCP and the controls

\begin{tabular}{|c|c|c|c|c|c|c|c|c|c|c|c|c|c|c|c|}
\hline & \multirow[b]{2}{*}{ No. } & \multirow[b]{2}{*}{ Source } & \multirow[b]{2}{*}{ df } & \multicolumn{3}{|c|}{ FA } & \multicolumn{3}{|c|}{ MD } & \multicolumn{3}{|c|}{$A D$} & \multicolumn{3}{|c|}{ RD } \\
\hline & & & & SS & F Value & $P^{a}$ & SS & F Value & $P^{a}$ & SS & F Value & $P^{a}$ & SS & F Value & $P^{a}$ \\
\hline \multirow[t]{3}{*}{ gCC } & \multirow[t]{3}{*}{30} & Group, & 1 & 0.083 & 14.43 & .0025 & 0.23 & 17.13 & .0012 & 0.037 & 1.6 & NS & 0.506 & 14.35 & .0025 \\
\hline & & age, & 1 & 0.184 & 31.73 & $<.0001$ & 0.515 & 38.26 & $<.0001$ & 0.097 & 4.16 & NS & 0.433 & 12.28 & .0041 \\
\hline & & scanner & 2 & 0.025 & 2.19 & NS & 0.025 & 0.94 & NS & 0.078 & 1.67 & NS & 0.026 & 0.37 & NS \\
\hline \multirow[t]{3}{*}{$\mathrm{sCC}$} & \multirow[t]{3}{*}{26} & Group, & 1 & 0.058 & 16.64 & .0017 & 0.079 & 5.42 & .06 & 0.001 & 0.02 & NS & 0.183 & 15.08 & .0025 \\
\hline & & age, & 1 & 0.2 & 57.69 & $<.0001$ & 0.98 & 67.25 & $<.0001$ & 0.56 & 10.98 & .0075 & 1.265 & 104.23 & $<.0001$ \\
\hline & & scanner & 2 & 0.005 & 0.73 & NS & 0.077 & 2.64 & NS & 0.137 & 1.34 & NS & 0.078 & 3.23 & NS \\
\hline \multirow[t]{3}{*}{ ALIC } & \multirow[t]{3}{*}{31} & Group, & 1 & 0.02 & 5.43 & .058 & 0.007 & 1.29 & NS & 0.076 & 12.48 & .004 & 0.000 & 0.00 & NS \\
\hline & & age, & 1 & 0.143 & 39.54 & $<.0001$ & 0.495 & 88.83 & $<.0001$ & 0.285 & 47.04 & $<.0001$ & 0.622 & 68.15 & $<.0001$ \\
\hline & & scanner & 2 & 0.002 & 0.31 & NS & 0.018 & 1.63 & NS & 0.015 & 1.28 & NS & 0.011 & 1.2 & NS \\
\hline \multirow[t]{3}{*}{ PLIC } & \multirow[t]{3}{*}{31} & Group, & 1 & 0.007 & 0.7 & NS & 0.018 & 3.06 & NS & 0.102 & 9.74 & .0096 & 0.00 & 0.01 & NS \\
\hline & & age, & 1 & 0.126 & 12.4 & .004 & 0.327 & 55.77 & $<.0001$ & 0.166 & 15.74 & .0017 & 0.415 & 31.44 & $<.0001$ \\
\hline & & scanner & 2 & 0.002 & 0.12 & NS & 0.014 & 1.2 & NS & 0.05 & 2.36 & NS & 0.003 & 0.11 & NS \\
\hline
\end{tabular}

Note:-No. indicates number of observations; SS, sum of squares (type II); NS, not significant; HCP, hydrocephalus; df, degrees of freedom.

${ }^{a}$ All $P$ values are adjusted for multiple testing using a false discovery rate method.

Table 2: Group comparison of ABAS-II scores

\begin{tabular}{llcrccc}
\hline & Group & No. & \multicolumn{1}{c}{ Mean } & $\boldsymbol{T}$ & df & $\boldsymbol{P}$ \\
\hline GAC & HCP, & 15 & $82.5 \pm 15.8$ & 3.64 & 29 & .001 \\
& control & 16 & $103.4 \pm 16.1$ & & & \\
Conceptual & HCP, & 15 & $84.7 \pm 15.9$ & 3.56 & 28 & .001 \\
& control & 15 & $105.5 \pm 16.1$ & & & \\
Practical & HCP, & 16 & $90.8 \pm 14.1$ & 2.10 & 29 & .044 \\
& control & 15 & $101.6 \pm 14.6$ & & & \\
Social & HCP, & 16 & $85.0 \pm 13.0$ & 2.41 & 29 & .026 \\
& control & 15 & $99.7 \pm 20.3$ & & & \\
Motor & HCP, & 17 & $88.8 \pm 17.0$ & 2.85 & 31 & .008 \\
& control & 16 & $104.7 \pm 14.5$ & & & \\
\hline
\end{tabular}

Note:- HCP indicates hydrocephalus; df, degrees of freedom; GAC, General Adaptive Composite.

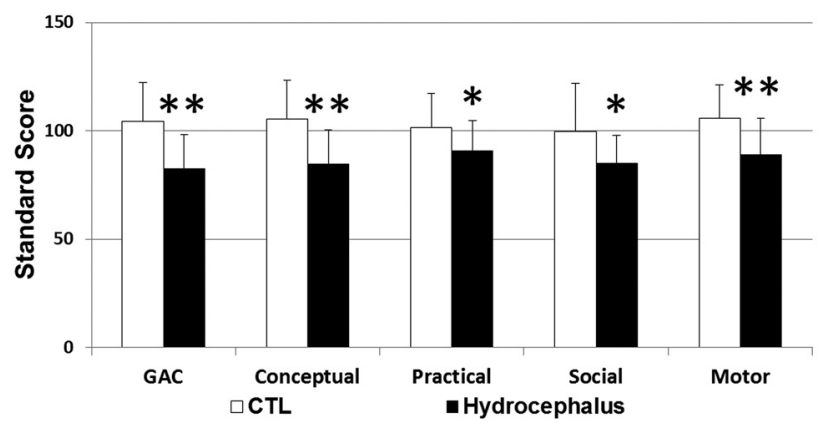

FIG 5. Comparison of neuropsychological results between control subjects and the children with hydrocephalus. The asterisk indicates a statistically significant group difference at $P<.05$; double asterisks, a statistically significant group difference at $P<.01$.

and $\mathrm{SCC}$, driven by the increase of RD and the relatively unchanged $\mathrm{AD}$. In contrast, in the PLIC and ALIC, many patients with hydrocephalus were found to have abnormally high FA values, while the remaining patients had abnormally low FA values. Finally, moderate-to-strong correlations were found between DTI indices and parent-reported motor skills.

DTI has been used to study various diseases and neurologic disorders in both clinical and basic experimental research. The characteristics of diffusion properties and the patterns of abnormality vary in different situations. Similar results have been found in some previous studies. ${ }^{9,11,12,23,24}$ In DWI studies, hydrocephalus was often found to be associated with abnormally high ADC values in periventricular white matter. ${ }^{23-25}$ In DTI

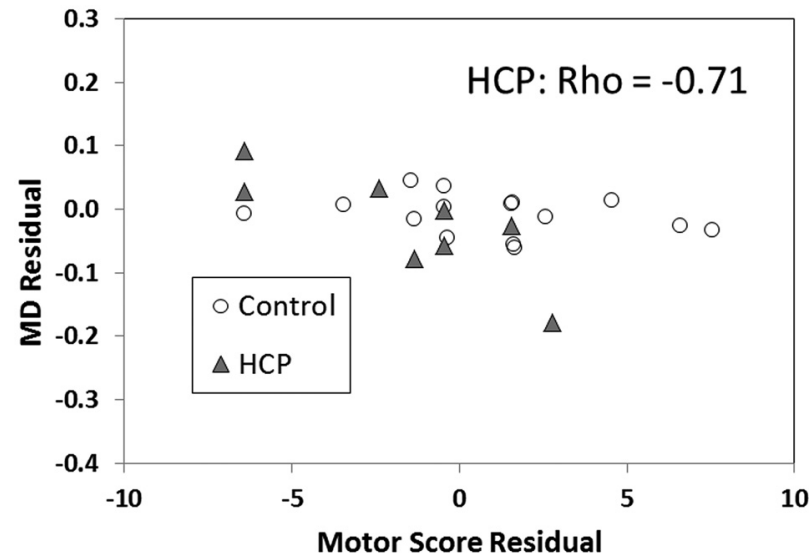

FIG 6. Correlation between DTI parameters in PLIC and the Motor scores based on ABAS-II parent report form.

studies, lower FA values have been consistently found in the corpus callosum in hydrocephalus, while mixed FA changes have been reported in some other white matter structures, such as the internal capsule. ${ }^{9-12}$

There have been extensive discussions on the interpretation of different abnormality patterns and the ability of DTI to derive information about underlying cellular changes. The observations reported in the present study fit well with some of these scenarios. In general, impairment in myelination and axonal damage are often associated with decreased FA and increased MD but are differentiated by an increase of $\mathrm{RD}$ (eg, in retinal ischemia) or $\mathrm{AD}$ (eg, in multiple sclerosis). ${ }^{26-28}$ Increased FA accompanied by decreased RD or increased AD or both is often regarded as the result of a compression of white matter tracts, such as results from space-occupying lesions. According to these general themes, the abnormalities seen in hydrocephalus may be attributed to various different underlying injury mechanisms. The decreased FA and increased MD and RD seen in the corpus callosum in our patient population are likely due to delayed or abnormal myelination. ${ }^{26,27}$ Similar patterns of abnormality have sometimes been regarded as a reflection of white matter damage that is difficult to recover. ${ }^{29}$ On the other hand, the increased FA in the internal capsule in many of our patients with hydrocephalus may suggest that in these areas, hydrocephalus causes increased homogeneity 
in fiber orientation. These changes can be attributed to mechanical compression, often regarded as benign and likely reversible after ventriculomegaly has been reduced by CSF drainage.

Although the group difference in RD was consistent in all 4 ROIs when younger participants were examined, the difference seemed to persist throughout the age range studied in gCC and sCC (Figs 1 and 2) but not in PLIC and ALIC. Attributing the changes to delay in myelination may be confounded by the development stage, at least for some brain regions, which should remain a topic for further investigation. On the other hand, in both the PLIC and ALIC, while many older children with hydrocephalus had higher FA, some very young patients had lower FA and higher $\mathrm{MD}, \mathrm{AD}$, and/or $\mathrm{RD}$ compared with the control group (Figs 3 and 4). There appears to be a potential bifurcation in the pattern of diffusion abnormality in patients with hydrocephalus. However, it is difficult to subcategorize the patient group with an objective criterion to further study the 2 patterns. We cannot rule out the possibility that bifurcation in the pattern of diffusion abnormality represents a limitation of our ability to draw accurate ROIs in the youngest age group, which contributes to the larger spread in values of the FA in that plot.

\section{Neuropsychological Deficits in Hydrocephalus and the Correlation with DTI}

In this report, we present a prospective DTI study examining the correlation of DTI parameters with neuropsychological deficits in surgical patients with pediatric hydrocephalus. The lack of such data in the literature is perhaps logistic; most notably, the narrow window between the time of diagnosis and surgical treatment often does not allow a comprehensive preoperative neuropsychological evaluation. It has been well documented that the most prevalent functional deficits in children with hydrocephalus are in visuospatial and motor domains. ${ }^{30}$ However, given the young age of the patients recruited for this project (between birth and approximately 51 months), the measures selected for this study were limited to broader developmental tests. Our ongoing long-term project includes formal infant testing by using the Bayley Scale of Infant Development-III, third edition. However, the current study looked solely at initial parent ratings on the ABAS-II, a test selected as an age-appropriate caregiver-report measure that can be carried out consistently across all ages throughout the study. It was chosen to provide insight into potential neurodevelopmental and functional deficits accompanying hydrocephalus and to assess the predictive power of DTI for outcome measures at various stages of hydrocephalus. Because of the small sample size and the non-normal distribution of the data, we used a nonparametric estimate of the correlation coefficient to represent this relationship. The correlation analysis has demonstrated moderate-tostrong associations between parent estimates of motor skill development and DTI indices of white matter integrity. Specifically, lower motor scores in the hydrocephalus group were negatively correlated with higher MD and RD in the PLIC. This can be interpreted as initial evidence for an association between functional deficits and underlying white matter alteration in our patient population. A larger sample size will be needed for more comprehensive modeling of the relation between the 2 domains. In addition, longitudinal data are needed to assess the correlation be- tween DTI and cognitive and adaptive outcome data at various points in time, from immediately postoperative to preschool and school-age so that we can better meet the needs of children as they become more independent.

\section{Limitation of the Study}

The participants in the control group were recruited from patients who were initially referred for clinical MR imaging but whose scan findings were negative. This was mainly due to the difficulty expected in acquiring good-quality DTI data in nonsedated, ageappropriate, healthy children. Although stringent criteria were adopted in the recruitment, it is still possible that these criteria will pose a potential confounding factor for the interpretation of our results. Another limitation arises from the region of interestbased DTI data analysis method used in the study. The severity and variability of ventriculomegaly seen in our patient population combined with developmental changes exclude the possibility of registering brain images to a common template for voxelwise group comparison. The major white matter structures, such as the corpus callosum and internal capsule, can still be delineated reliably in hydrocephalic brains. However, it would pose significant technical difficulty if used in other structures that might also be vulnerable to hydrocephalus (eg, frontal lobe white matter regions). An additional limitation comes from the age and scanner differences in the 2 groups. Although effort has been made in the data analysis to remove the age and scanner effect, it is possible that our initial results were influenced by the heterogeneity in these 2 factors.

\section{CONCLUSIONS}

We demonstrated that the DTI indices derived from the prospectively acquired data are significantly affected in white matter structures in children with hydrocephalus at the acute stage, before surgical treatment. The various patterns of DTI abnormalities in the corpus callosum and internal capsule suggest regionspecific multifactorial variation in underlying white matter injury mechanisms. In neuropsychological tests, parents of children with hydrocephalus endorsed significant developmental deficits, in which the motor scores were found to correlate with DTI measures in functionally relevant white matter structures. As further data are collected longitudinally, we will quantify the progression of white matter structural changes and assess their clinical relevance to both short- and long-term surgical outcomes.

Disclosures: Robert C. McKinstry—UNRELATED: Consultancy: Siemens, Comments: I was paid as an actor in a Siemens TV commercial "Somewhere in America," Payment for Lectures (including Speakers Bureaus): Siemens, Comments: Siemens will be flying me to a meeting to present unrelated research using the new PET/MRI scanner, Other: Siemens, Comments: Siemens will be flying me to Germany to tour the factory and talk with engineers and product managers about future product innovations. Joshua Shimony—RELATED: Grant: NIH. Jannel Phillips—RELATED: Grant: $\mathrm{NIH},{ }^{*}$ Comments: The case was part of a larger multicenter study looking at outcomes of hydrocephalus. David Limbrick—RELATED: Grant: NIH/NINDS, ${ }^{*}$ Comments: Grant: longitudinal DTI study in children treated for congenital hydrocephalus (R01 NS066932). Scott Holland-RELATED: Grant: NIH, * Comments: This work was supported by a grant from NINDS R01 NS066932 to the principal investigator, W. Yuan, Support for Travel to Meetings for the Study or Other Purposes: NIH, ${ }^{*} \mathrm{Com}-$ ments: This work was supported by a grant from NINDS R01 NS066932 to the principal investigators, W. Yuan and F.T. Mangano. Mekibib Altaye-RELATED: Grant: $\mathrm{NIH},{ }^{*}$ Comments: I have a percentage time effort on this grant that is paid to me as part of my salary from the institution. Sarah Simpson-RELATED: NIH, Comments: 
My salary comes from the grant from NIH that supports this research. Deanna Mercer-RELATED: Grant: NIH/NINDS grant R01NS066932-03. *Money paid to the institution.

\section{REFERENCES}

1. Del Bigio MR, Wilson MJ, Enno T. Chronic hydrocephalus in rats and humans: white matter loss and behavior changes. Ann Neurol 2003;53:337-46

2. Del Bigio MR, Zhang YW. Cell death, axonal damage, and cell birth in the immature rat brain following induction of hydrocephalus. Exp Neurol 1998;154:57-69

3. Fletcher JM, Bohan TP, Brandt ME, et al. Cerebral white matter and cognition in hydrocephalic children. Arch Neurol 1992;49:818-24

4. Fletcher JM, McCauley SR, Brandt ME, et al. Regional brain tissue composition in children with hydrocephalus: relationships with cognitive development. Arch Neurol 1996;53:549-57

5. Hannay HJ. Functioning of the corpus callosum in children with early hydrocephalus. J Int Neuropsychol Soc 2000;6:351-61

6. Khan $\mathrm{OH}$, Enno TL, Del Bigio MR. Brain damage in neonatal rats following kaolin induction of hydrocephalus. Exp Neurol 2006;200:311-20

7. Zimmerman RD, Fleming CA, Lee BC, et al. Periventricular hyperintensity as seen by magnetic resonance: prevalence and significance. AJR Am J Roentgenol 1986;146:443-50

8. Bradley WG Jr. MR prediction of shunt response in NPH: CSF morphology versus physiology. AJNR Am J Neuroradiol 1998;19: 1285-86

9. Assaf Y, Ben-Sira L, Constantini S, et al. Diffusion tensor imaging in hydrocephalus: initial experience. AJNR Am J Neuroradiol 2006;27:1717-24

10. Hasan KM, Eluvathingal TJ, Kramer LA, et al. White matter microstructural abnormalities in children with spina bifida myelomeningocele and hydrocephalus: a diffusion tensor tractography study of the association pathways. J Magn Reson Imaging 2008;27:700-09

11. Herweh C, Akbar M, Wengenroth M, et al. DTI of commissural fibers in patients with Chiari II-malformation. Neuroimage 2009;44: 306-11

12. Yuan W, Mangano FT, Air EL, et al. Anisotropic diffusion properties in infants with hydrocephalus: a diffusion tensor imaging study. AJNR Am J Neuroradiol 2009;30:1792-98

13. Air EL, Yuan W, Holland SK, et al. Longitudinal comparison of preand postoperative diffusion tensor imaging parameters in young children with hydrocephalus. J Neurosurg Pediatr 2010;5:385-91

14. Harrison PL, Oakland T. Adaptive Behavior Assessment System. 2nd ed, San Antonio, Texas: Harcourt Assessment; 2003

15. Jiang H, van Zijl PC, Kim J, et al. DTIStudio: resource program for diffusion tensor computation and fiber bundle tracking. Comput Methods Programs Biomed 2006;81:106-16

16. Woods RP, Grafton ST, Watson JD, et al. Automated image registration. II. Intersubject validation of linear and nonlinear models. J Comput Assist Tomogr 1998;22:153-65

17. Basser PJ, Pierpaoli C. A simplified method to measure the diffusion tensor from seven MR images. Magn Reson Med 1998;39:928-34

18. Hermoye L, Saint-Martin C, Cosnard G, et al. Pediatric diffusion tensor imaging: normal database and observation of the white matter maturation in early childhood. Neuroimage 2006;15 29:493-504

19. Yuan W HS, Shimony JS, Jones BV, et al. Quality assurance in multiinstitution and multi-scanner MRI neuroimaging research. In: Proceedings of the 6th Congress and Exhibition of the Joint Societies of Paediatric Radiology, London, UK. May 27-31, 2011

20. Kruggel F, Turner J, Muftuler LT. Impact of scanner hardware and imaging protocol on image quality and compartment volume precision in the ADNI cohort. Neuroimage 2010; 49:2123-33

21. Colombo P, Baldassarri A, Del Corona M, et al. Multicenter trial for the set-up of a MRI quality assurance programme. Magn Reson Imaging 2004;22:93-101

22. Van Horn JD, Toga AW. Multisite neuroimaging trials. Current Opin Neurol 2009;22:370-78

23. Uluğ AM, Truong TN, Filippi CG, et al. Diffusion imaging in obstructive hydrocephalus. AJNR Am J Neuroradiol 2003;24:1171-76

24. Leliefeld PH, Gooskens RH, Tulleken CA, et al. Noninvasive detection of the distinction between progressive and compensated hydrocephalus in infants: is it possible? J Neurosurg Pediatr 2010;5:562-68

25. Del Bigio MR, Slobodian I, Schellenberg AE, et al. Magnetic resonance imaging indicators of blood-brain barrier and brain water changes in young rats with kaolin-induced hydrocephalus. Fluids Barriers CNS 2011;8:22

26. Song SK, Sun SW, Ramsbottom MJ, et al. Dysmyelination revealed through MRI as increased radial (but unchanged axial) diffusion of water. Neuroimage 2002;17:1429-36

27. Song SK, Yoshino J, Le TQ, et al. Demyelination increases radial diffusivity in corpus callosum of mouse brain. Neuroimage 2005;26:132-40

28. Budde MD, Kim JH, Liang HF, et al. Axonal injury detected by in vivo diffusion tensor imaging correlates with neurological disability in a mouse model of multiple sclerosis. NMR Biomed 2008;21:589-97

29. Goebell E, Paustenbach S, Vaeterlein O, et al. Low-grade and anaplastic gliomas: differences in architecture evaluated with diffusion-tensor MR imaging. Radiology 2006;239:217-22

30. Mataró M, Junque C, Poca MA, et al. Neuropsychological findings in congenital and acquired childhood hydrocephalus. Neuropsychol Rev 2001;11:169-78 